\title{
Early discharge compared with ordinary discharge after percutaneous coronary intervention - a systematic review and meta-analysis of safety and cost
}

This article was published in the following Dove Press journal:

Vascular Health and Risk Management

20 March 2017

Number of times this article has been viewed

\author{
Michael Abdelnoor ${ }^{1,2}$ \\ Jack Gunnar Andersen ${ }^{3,4}$ \\ Harald Arnesen ${ }^{2,5}$ \\ Odd Johansen ${ }^{6}$ \\ 'Oslo Centre of Biostatistics and \\ Epidemiology, Oslo University \\ Hospital, Oslo, Norway; ${ }^{2}$ Center for \\ Clinical Heart Research, Department \\ of Cardiology, Oslo University \\ Hospital, Oslo, Norway; ${ }^{3} \mathrm{Clinic}$ of \\ Radiology and Nuclear Medicine, Oslo \\ University Hospital, Oslo, Norway; \\ ${ }^{4}$ Department of Health Management \\ and Health Economics, Faculty of \\ Medicine, University of Oslo, Oslo, \\ Norway; ${ }^{5}$ Faculty of Medicine, \\ University of Oslo, Oslo Norway; \\ ${ }^{6}$ Department of Cardiology, Oslo \\ University Hospital, Oslo, Norway
}

Correspondence: Jack Gunnar Andersen Clinic of Radiology and Nuclear Medicine, Oslo University Hospital, Postboks 4956 Nydalen, 0424 Oslo, Norway

Tel +479864l 634

Email uxjaga@ous-hf.no

\begin{abstract}
Aim: We aimed to summarize the pooled effect of early discharge compared with ordinary discharge after percutaneous coronary intervention (PCI) on the composite endpoint of reinfarction, revascularization, stroke, death, and incidence of rehospitalization. We also aimed to compare costs for the two strategies.

Methods: The study was a systematic review and a meta-analysis of 12 randomized controlled trials including 2962 patients, followed by trial sequential analysis. An estimation of cost was considered. Follow-up time was 30 days.

Results: For early discharge, pooled effect for the composite endpoint was relative risk of efficacy (RRe) $=0.65,95 \%$ confidence interval $(\mathrm{CI})(0.52-0.81)$. Rehospitalization had a pooled effect of $\mathrm{RRe}=1.10,95 \% \mathrm{CI}(0.88-1.38)$. Early discharge had an increasing risk of rehospitalization with increasing frequency of hypertension for all populations, except those with stable angina, where a decreasing risk was noted. Advancing age gave increased risk of revascularization. Early discharge had a cost reduction of 655 Euros per patient compared with ordinary discharge. Conclusion: The pooled effect supports the safe use of early discharge after PCI in the treatment of a heterogeneous population of patients with coronary artery disease. There was an increased risk of rehospitalization for all subpopulations, except patients with stable angina. Clinical trials with homogeneous populations of acute coronary syndrome are needed to be conclusive on this issue.
\end{abstract}

Keywords: percutaneous coronary intervention, early discharge, cost, meta-analysis

\section{Introduction}

Today, percutaneous coronary intervention (PCI) is one of the most commonly performed cardiac interventions. ${ }^{1}$ The cost of treatment for acute coronary syndrome (ACS) in Western Europe is high and constitutes a considerable portion of total health care expenses. This is mainly attributed to revascularization procedures and the cost of hospital stay. ${ }^{2,3}$

Systematic use of stents and potent platelet inhibitors has revolutionized the success rate after PCI by drastically reducing the risk of acute occlusion in the first 24 hours after a successful procedure. ${ }^{4}$ Reduced catheter sizes have made a transradial approach more feasible, which in turn has reduced bleeding incidents as well as the necessary observation time. Early discharge is routinely practiced in some parts of Europe and Canada but is rare in the US. Reasons may be concerns over patient safety and 
short-term clinical events, differences in procedures and use of pharmacology, as well as different systems for financial compensation.

With an increasing demand for hospital resources, the trend has moved toward the implementation of fast-track treatment pathways. ${ }^{5}$ Several observational studies, and some randomized controlled trials (RCTs), have aimed to prove that same-day or early discharge after PCI is both feasible and safe. The patient populations in these studies include those with elective PCI, unstable angina pectoris (UAP), non-ST elevation myocardial infarction (NSTEMI), and ST elevation myocardial infarction (STEMI) patients. ${ }^{6,7}$

Cost analyses of early discharge versus ordinary discharge show that early discharge is economically favorable, primarily by eliminating the cost of overnight observation. ${ }^{8-10}$

Two meta-analyses and systematic reviews ${ }^{11,12}$ claimed, based on observational studies and RCTs, support of programs for same-day discharge after PCI for selected groups of patients without defining the groups. The study populations in the RCTs that have been executed in this field are all relatively small. We have undertaken a systematic review and meta-analysis of only RCTs and have not considered observational studies.

The aim of this study was to summarize the pooled effect of early discharge compared with ordinary discharge after PCI on the primary composite endpoint of re-infarction, revascularization, stroke, death, and the incidence of rehospitalization. Second, we wanted to compare costs for the two strategies.

\section{Methods}

We searched the Cochrane Central Register of controlled trials (1970-2015), Embase (1980-2015), and Medline/ PubMed (1966-2015). We combined the keywords outpatient, same day discharge, early discharge, percutaneous coronary intervention, PCI, angioplasty, economy, and cost. Similar strategies were used to search for previously published metaanalyses and reviews. We also screened reference lists and contacted authors. Our last update for research was done on August 5, 2015. No limitation on language was considered.

Inclusion criteria for the studies were RCTs comparing early discharge versus ordinary discharge after PCI and safety outcome data for up to 30 days following the index intervention, available for both groups separately. We included only studies from 1999 onward.

When cost analysis was available, it had to include an overview of cost related to both groups separately.
Twelve RCTs were considered eligible for this study (Figure 1). ${ }^{4,13-23}$

The definition of early discharge differs between the studies because of different patient populations. Eight studies have patients with stable coronary artery disease (CAD), UAP, and NSTEMI. ${ }^{4,15-18,20,22,23}$ They define early discharge as discharge on the same day as the intervention. The remaining four studies have patients with STEMI. ${ }^{13,14,19,21}$ Their definition of early discharge was discharge between 48 and 72 hours after the intervention.

Three of these included an analysis of cost. ${ }^{20,22,23}$ Two additional articles with an assessment of cost were based on studies already included in the safety analysis, and we added these to our cost analyses. ${ }^{8,9}$ Andersen et a ${ }^{16}$ originally included 399 patients in their study. In the meta-analysis of safety, we have considered only the 167 who had PCI during their index stay.

\section{Endpoints}

Our primary endpoint was the incidence of the composite cardiovascular events mortality, re-infarction, revascularization, and stroke. The secondary endpoint was rehospitalization, both endpoints within 30 days after PCI. Finally, a 30-day estimation of the cost of the two strategies was considered.

The statistical methods used for our analyses have previously been described in detail by Abdelnoor et al. ${ }^{24}$

Trial sequential analysis (TSA) was used according to Pogue and Yusuf ${ }^{25}$ and Wetterslev et al. ${ }^{26}$ We used TSA as it is implemented in the STATA Program 12 (StataCorp LLC, College Station, TX, USA). This method permits to consider the accumulated number of patients to estimate the power of our cumulative meta-analysis.

Robustness of the pooled estimates was checked by sensitivity analysis. Each of the studies was individually omitted from the data set, followed in each case by recalculation of the pooled estimate of the remaining studies.

We followed the PRISMA guidelines for meta-analyses and systematic reviews of randomized trials in this report. ${ }^{27}$

\section{Results}

The baseline characteristics are represented in Table 1. The 12 trials included 2962 patients, 1486 patients in the early discharge group and 1476 patients in the ordinary discharge group. Considering the study-level variables with regard to the primary endpoint, $37 \%$ had concealment of randomization, $27 \%$ had outcome blinded to the investigators, $91 \%$ 
Medline/PubMed

Embase

Cochrane Central Register of Controlled Trials

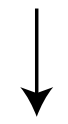

756 references identified

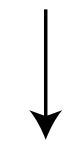

90 RCTs

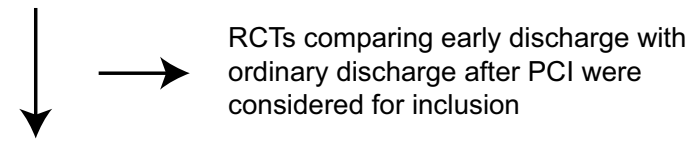

13 potentially eligible

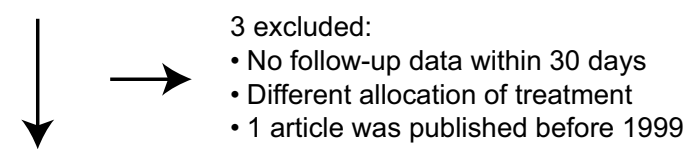

10 references from search

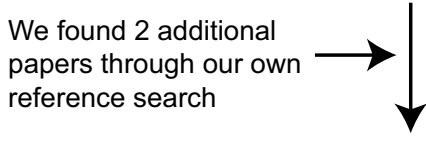

12 studies included in the meta-analysis

Figure I Details of the search process. Last search was performed August 5, 2015.

Abbreviations: RCTs, randomized controlled trials; $\mathrm{PCl}$, percutaneous coronary intervention.

Table I Baseline characteristics

\begin{tabular}{|c|c|c|c|c|c|c|c|c|c|c|c|c|}
\hline \multirow[t]{2}{*}{ Characteristics } & \multicolumn{12}{|c|}{ References } \\
\hline & $\begin{array}{l}\text { Azzalini } \\
\text { et } \mathrm{al}^{13} \\
(\mathrm{n}=100)\end{array}$ & $\begin{array}{l}\text { Melberg } \\
\text { et } \mathrm{al}^{14} \\
(\mathrm{n}=2 \mid 5)\end{array}$ & $\begin{array}{l}\text { Kim } \\
\text { et } \mathrm{al}^{15} \\
(\mathrm{n}=298)\end{array}$ & $\begin{array}{l}\text { Falcone } \\
\text { et } \mathrm{al}^{17} \\
(\mathrm{n}=44)\end{array}$ & $\begin{array}{l}\text { Bertrand } \\
\text { et }\left.a\right|^{18} \\
(n=\mid 005)\end{array}$ & $\begin{array}{l}\text { Kotowycz } \\
\text { et al }{ }^{19} \\
(n=54)\end{array}$ & $\begin{array}{l}\text { Glaser } \\
\text { et } \mathrm{al}^{20} \\
(\mathrm{n}=39)\end{array}$ & $\begin{array}{l}\text { Jirmar } \\
\text { et } \mathrm{al}^{21} \\
(\mathrm{n}=56)\end{array}$ & $\begin{array}{l}\text { Heyde } \\
\text { et } \mathrm{al}^{22} \\
(\mathrm{n}=800)\end{array}$ & $\begin{array}{l}\text { Carere } \\
\text { et } \mathrm{al}^{23} \\
(\mathrm{n}=\mid 00)\end{array}$ & $\begin{array}{l}\text { Andersen } \\
\text { et } \mathrm{a}^{16} \\
(\mathrm{n}=167)\end{array}$ & $\begin{array}{l}\text { Knopf } \\
\text { et } \mathrm{al}^{4} \\
(\mathrm{n}=90)\end{array}$ \\
\hline UAP, n & 0 & 0 & 0 & 0 & 482 & 0 & 0 & 0 & 75 & 50 & 34 & 38 \\
\hline NSTEMI, n & 0 & 0 & 0 & 0 & 188 & 0 & 0 & 0 & 0 & 0 & 133 & 0 \\
\hline STEMI, $\mathrm{n}$ & 100 & 215 & 0 & 0 & 335 & 54 & 0 & 56 & 0 & 0 & 0 & 0 \\
\hline Stable AP, n & 0 & 0 & 298 & 44 & 0 & 0 & 39 & 0 & 725 & 50 & 0 & 52 \\
\hline Age, mean & 59.2 & - & 55.9 & 59 & 60.5 & 55.3 & 61 & 56.7 & 61.6 & 60.5 & 66 & 58 \\
\hline Female \% & 14 & 13.5 & 26 & 29.5 & 21.4 & 25.9 & 5 & $4 I .1$ & 19 & 17 & 25 & 37 \\
\hline Diabetes \% & 12 & 7.9 & 49.3 & 45.5 & 16 & 22.5 & 33 & 23.4 & 15 & - & 22 & 20 \\
\hline Hypertension \% & 44 & 29.8 & - & 90.9 & 53.5 & 44.5 & 77 & 41.2 & 40 & - & 53 & 56 \\
\hline Prior MI \% & 4 & 6.5 & - & 25 & 44.1 & - & 31 & 0 & 36 & - & 27 & 31 \\
\hline Prior $\mathrm{PCl} \%$ & 4 & 5.6 & - & 45.4 & 19.9 & - & 56 & - & 21 & - & 34 & 47 \\
\hline Prior CABG \% & 0 & 0.5 & - & - & - & - & 10 & - & 4 & - & 8 & 13 \\
\hline Femoral access \% & 9 & $4 I .4$ & 66.4 & 100 & 0 & - & 100 & 100 & 100 & 100 & 11.8 & 100 \\
\hline
\end{tabular}

Abbreviations: -, unknown; UAP, unstable angina pectoris; NSTEMI, non-ST elevation myocardial infarction; STEMI, ST elevation myocardial infarction; AP, angina pectoris; prior MI, prior myocardial infarction; prior PCl, prior percutaneous coronary intervention; prior CABG, prior coronary artery bypass graft.

used the intention-to-treat analysis, and $81 \%$ of the trials did not have drop-out during follow-up. For the outcome rehospitalization, $36 \%$ had concealment of randomization, $27 \%$ had blinding of the investigators to the outcome, $91 \%$ used the intention-to-treat analysis, and $82 \%$ of the trials did not have drop-out during follow-up.

The majority of the trials suffered serious methodological biases and were power deficient. Five RCTs included 
cost analysis and were included in the cost study. Three of these had correct concealment, one had blinding of investigator to the outcome, four followed the intentionto-treat analysis, and three had no drop-out during followup. All costs were recalculated to the value of the Euro in December 2014.

\section{Primary composite endpoint}

The pooled estimate of the efficacy (Tables 2 and 3 ) showed an estimate of relative risk $[\mathrm{RR}]=0.65,95 \%$ confidence interval $(95 \% \mathrm{CI})(0.52-0.81), p=0.0002$, indicating a lower risk in the early discharge group compared to ordinary discharge group for the primary composite endpoint with no heterogeneity. There was no selection bias. We ran subgroup and metaregression analyses on study-level and patient-level variables. Meta-regression is a method to investigate heterogeneity, and it permits to quantify the association between variables and effect of the intervention. With regard to study-level variables, none was associated with the efficacy. Considering patientlevel variables, none of the covariates showed an association with the efficacy on the primary endpoint (Tables 2 and 3).

A sensitivity analysis revealed the non-robustness of our results for the primary composite endpoint. Our hypothesis was a risk reduction of $15 \%$ lower incidence in the ordinary care group versus early discharge group. For an accumulated number of 2912 patients and a type I error of 5\%, we have a power of the cumulative meta-analysis of $98 \%$. For the primary composite endpoint, our meta-analysis includes enough patients and has satisfactory power.

\section{Re-infarction and rehospitalization}

Another run of pooled efficacy and sensitivity analysis on re-infarction and incidence of revascularization showed that for re-infarction the pooled RR of efficacy

Table 2 Pooled estimate of RR of efficacy for ED compared to $\mathrm{OC}$ on the primary composite endpoint, with stratification on attrition bias and detection bias using the fixed-effect model

\begin{tabular}{|c|c|c|c|c|c|}
\hline Subdivision & Studies (n) & $\operatorname{RRe}(95 \% \mathrm{Cl})$ & $\mathrm{Tau}^{2}$ & $I^{2}, \%$ & $p$-value ${ }^{a}$ \\
\hline All studies & 11 & $0.65(0.52-0.8 I)$ & 0.00 & 0.0 & 0.0002 \\
\hline \multicolumn{6}{|l|}{ No drop-out } \\
\hline Yes & 9 & $0.73(0.46-1.18)$ & 0.0000 & 0.0 & $0.558 I$ \\
\hline No/unclear & 2 & $0.73(0.46-1.18)$ & 0.0001 & 0.0 & \\
\hline \multicolumn{6}{|c|}{ Investigator blind to the outcome } \\
\hline Yes & 2 & $0.62(0.48-0.80)$ & 0.0000 & 0.0 & 0.4785 \\
\hline No & 9 & $0.75(0.47-1.18)$ & 0.000 & 0.0 & \\
\hline
\end{tabular}

Notes: $\mathrm{Tau}^{2}$ : inter-study variability. ${ }^{\mathrm{a}}$ Test for subgroup differences using randomeffect model between groups with $Q$ statistics $d f=I$ and $p$-value. Data adapted from references 4, 13-18, and 20-23.

Abbreviations: RRe, relative risk of efficacy; $E D$, early discharge; OC, ordinary care; $\mathrm{Cl}$, confidence interval.
Table 3 Estimate of the mixed-effect regression model between logRR and primary composite endpoint and the different studylevel and patient-level variables in a univariate analysis using II trials

\begin{tabular}{|c|c|c|c|c|c|}
\hline Covariates & Level & $\beta$-coefficient & Std err $(\beta)$ & $\mathbf{Z}$ & $P$-value \\
\hline $\begin{array}{l}\text { Publication } \\
\text { year }\end{array}$ & Years & -0.0305 & 0.0886 & -0.34 & 0.7304 \\
\hline Concealment & Yes/no & 0.5152 & 0.9178 & 0.56 & 0.5745 \\
\hline $\begin{array}{l}\text { Blinding } \\
\text { investigator }\end{array}$ & Yes//no & -0.1885 & 0.2659 & -0.70 & 0.4785 \\
\hline No drop-out & Yes/no & 0.1600 & 0.2731 & 0.58 & 0.5581 \\
\hline ITT analysis & Yes/no & -0.8105 & 0.8355 & 0.97 & 0.3320 \\
\hline $\begin{array}{l}\text { Age of the } \\
\text { patient }\end{array}$ & Years & 0.0363 & 0.1688 & 0.21 & 0.8298 \\
\hline Female & Percentage & 0.0179 & 0.0218 & 0.82 & 0.4100 \\
\hline Diabetes & Percentage & 0.0165 & $0.054 I$ & 0.30 & 0.7595 \\
\hline Hypertension & Percentage & 0.0167 & 0.0189 & 0.88 & 0.3744 \\
\hline STEMI & Percentage & 0.0019 & 0.0069 & 0.27 & 0.7838 \\
\hline $\begin{array}{l}\text { NSTEMI or } \\
\text { UAP }\end{array}$ & Percentage & 0.0039 & 0.0044 & 0.89 & 0.3739 \\
\hline Stable AP & Percentage & -0.0021 & 0.0039 & -0.17 & 0.4776 \\
\hline
\end{tabular}

Note: Data adapted from references 4, 13-18, and 20-23.

Abbreviations: RR, relative risk; Std err, standard error; ITT, intention-to-treat analysis; STEMI, ST elevation myocardial infarction; NSTEMI, non-ST elevation myocardial infarction; UAP, unstable angina pectoris; AP, angina pectoris.

was $\mathrm{RR}=0.45,95 \%$ CI $(0.31-0.66), p=0.001$, for early discharge compared to ordinary discharge, with no heterogeneity considering 10 studies. A sensitivity analysis confirmed the non-robustness of our results with regard to re-infarction. With regard to revascularization, we found a pooled efficacy of $\mathrm{RR}=0.38,95 \% \mathrm{CI}(0.18-0.79), p=0.009$, for early discharge compared to ordinary discharge. A sensitivity analysis pinpointed the non-robustness of our results. Meta-regression showed a significant increase of risk for revascularization in the early discharge group with increasing prevalence of NSTEMI/UAP (Figure 2) and the same with increasing age of the patient. With increasing prevalence of stable angina, there was a decreasing risk of revascularization in the early discharge group compared with ordinary discharge (Figure 3).

\section{Endpoint rehospitalization}

The pooled estimate of the effect of the early discharge showed an estimate of $\mathrm{RR}=1.10,95 \% \mathrm{CI}(0.88-1.38), p=0.37$, with a substantial heterogeneity of $16 \%$ with no difference of efficacy in the two groups, There was no selection bias. A sensitivity analysis using the random model indicated the non-robustness of our results. We ran a subgroup analysis (Table 4) and meta-regression (Table 5) on study-level and patient-level variables. Regarding study-level variables, concealment $(p=0.004)$, blinding $(p=0.001)$, and drop-out 


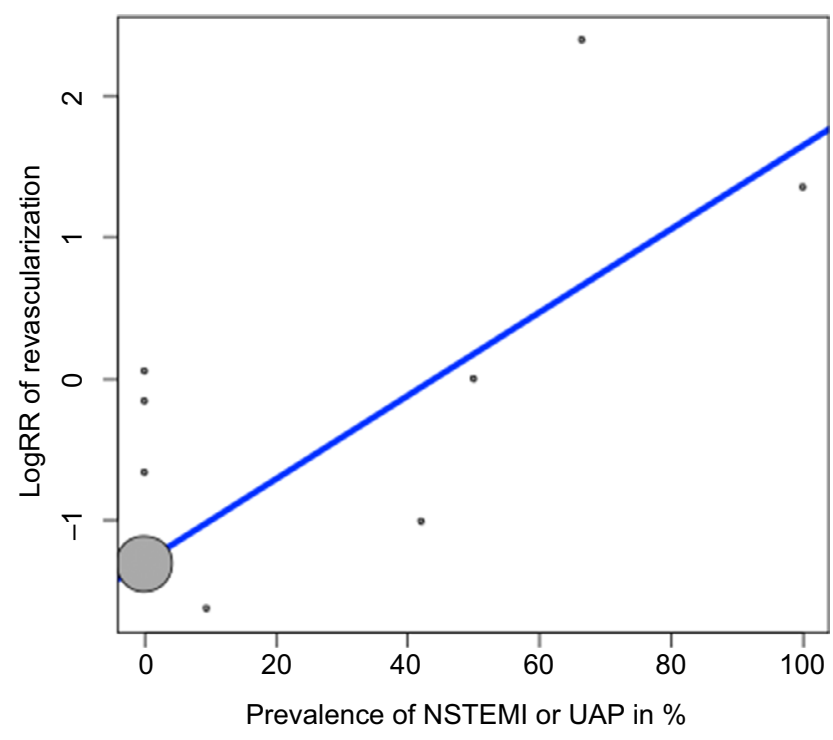

Figure 2 Meta-regression for the efficacy of ED compared to OD on the incidence of revascularization versus prevalence of NSTEMI or UAP

Notes: The size of the circles is an indicator of the size of the trial. Data adapted from references 4, 13-16, 18, 20, 21, and 23.

Abbreviations: ED, early discharge; OD, ordinary discharge; NSTEMI, non-ST elevation myocardial infarction; UAP, unstable angina pectoris; RR, relative risk.

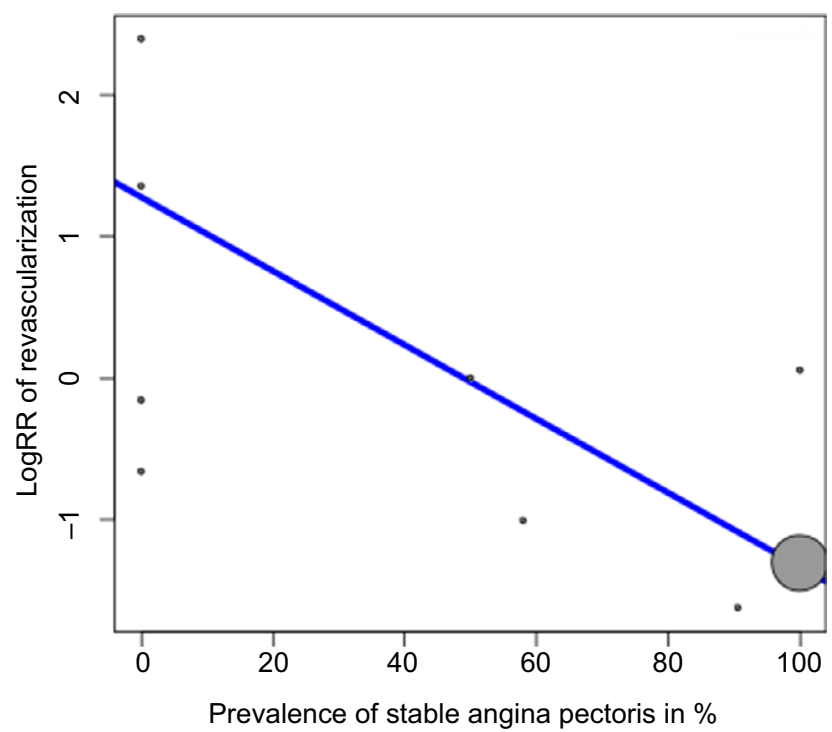

Figure 3 Meta-regression for the efficacy of ED compared to OD on the incidence of revascularization versus prevalence of stable angina pectoris.

Notes: The size of the circles is an indicator of the size of the trial. Data adapted from references 4, 13-16, 18, 20, 21, and 23.

Abbreviations: $E D$, early discharge; $O D$, ordinary discharge; $R R$, relative risk.

( $p=0.004)$ during follow-up were associated with efficacy, as also the prevalence of STEMI $(p=0.005)$, NSTEMI/UAP $(p=0.001)$, and stable angina $(p=0.008)$. This indicates an association between efficacy of early discharge and those variables.

For the patient-level variables, the frequency of hypertension was associated with efficacy $(p=0.004)$ (Figure 4$)$. This indicates increasing risk of rehospitalization for patients in the early discharge group compared to the
Table 4 Pooled estimate of RR of efficacy for ED compared to $O D$ on the endpoint rehospitalization, with stratification on concealment and no drop-out using the random-effect model

\begin{tabular}{|c|c|c|c|c|c|}
\hline Subdivision & Studies (n) & $\operatorname{RRe}(95 \% \mathrm{Cl})$ & $\mathrm{Tau}^{2}$ & $\mathbf{I}^{2}, \%$ & $p$-value ${ }^{a}$ \\
\hline All studies & 12 & $1.10(0.88-1.38)$ & 0.0191 & 16 & 0.3713 \\
\hline \multicolumn{6}{|l|}{ Concealment } \\
\hline Yes & 4 & $0.88(0.71-1.10)$ & 0 & 0 & 0.0039 \\
\hline No/Unclear & 8 & $1.34(1.13-1.61)$ & 0 & 0 & \\
\hline \multicolumn{6}{|c|}{ No drop-out during follow-up } \\
\hline Yes & 10 & $1.34(1.13-161)$ & 0.2382 & 21.6 & 0.5868 \\
\hline No & 2 & $\mathrm{I} .04(0.4 \mathrm{I}-2.6 \mathrm{I})$ & 0 & 0 & \\
\hline
\end{tabular}

Notes: $\mathrm{Tau}^{2}$ : inter-study variability. ${ }^{\mathrm{a}}$ Test for subgroup differences using randomeffect model between groups with $Q$ statistics $d f=I$ and $p$-value. Data adapted from references 4 and 13-23.

Abbreviations: RRe, relative risk of efficacy; ED, early discharge; OD, ordinary discharge; $\mathrm{Cl}$, confidence interval.

Table 5 Estimate of the mixed-effect regression model between $\log R R$ and rehospitalization and the different study-level and patient-level variables in a univariate analysis using 12 trials

\begin{tabular}{|c|c|c|c|c|c|}
\hline Covariates & Level & $\beta$-coefficient & Std err $(\beta)$ & $\mathbf{Z}$ & $p$-value \\
\hline $\begin{array}{l}\text { Publication } \\
\text { year }\end{array}$ & Years & -0.0178 & 0.0491 & -0.36 & 0.7170 \\
\hline Concealment & Yes/no & -0.4184 & 0.1449 & -2.88 & 0.0039 \\
\hline $\begin{array}{l}\text { Blinding } \\
\text { investigator }\end{array}$ & Yes/no & -0.4706 & $0.143 \mid$ & -3.28 & 0.0010 \\
\hline No drop-out & Yes/no & 0.4194 & 0.1451 & 2.89 & 0.0038 \\
\hline ITT analysis & Yes/no & -1.2674 & 1.3266 & -0.95 & 0.3339 \\
\hline $\begin{array}{l}\text { Age of the } \\
\text { patient }\end{array}$ & Years & 0.0596 & 0.0820 & 0.72 & 0.4674 \\
\hline Female & Percentage & 0.0189 & 0.0444 & 0.4259 & 0.6701 \\
\hline Diabetes & Percentage & -0.0084 & 0.0127 & -0.66 & 0.5091 \\
\hline Hypertension & Percentage & 0.0297 & 0.0102 & 2.91 & 0.0036 \\
\hline STEMI & Percentage & 0.0100 & 0.0036 & 2.81 & 0.005 \\
\hline $\begin{array}{l}\text { NSTEMI or } \\
\text { UAP }\end{array}$ & Percentage & 0.0079 & 0.0024 & 3.28 & 0.0010 \\
\hline Stable AP & Percentage & -0.0052 & 0.0016 & -3.25 & 0.008 \\
\hline
\end{tabular}

Note: Data adapted from references 4 and 13-23.

Abbreviations: RR, relative risk; Std err, standard error; ITT, intention-to-treat analysis; STEMI, ST elevation myocardial infarction; NSTEMI, non-ST elevation myocardial infarction; UAP, unstable angina pectoris; AP, angina pectoris.

ordinary care group with increasing frequency of hypertension, increasing prevalence of STEMI, and increasing prevalence of NSTEMI/UAP. There was a decreasing risk for rehospitalization with increasing prevalence of stable angina (Figure 5).

The TSA for the endpoint rehospitalization had a hypothesis of $15 \%$ risk reduction for rehospitalization in the ordinary discharge group compared to the early discharge group. For an accumulated number of 2912 patients and a type I error of $5 \%$, we have a power of the cumulative meta-analysis of $92 \%$.

We can conclude that for the endpoint rehospitalization, our cumulative meta-analysis has satisfactory power. 


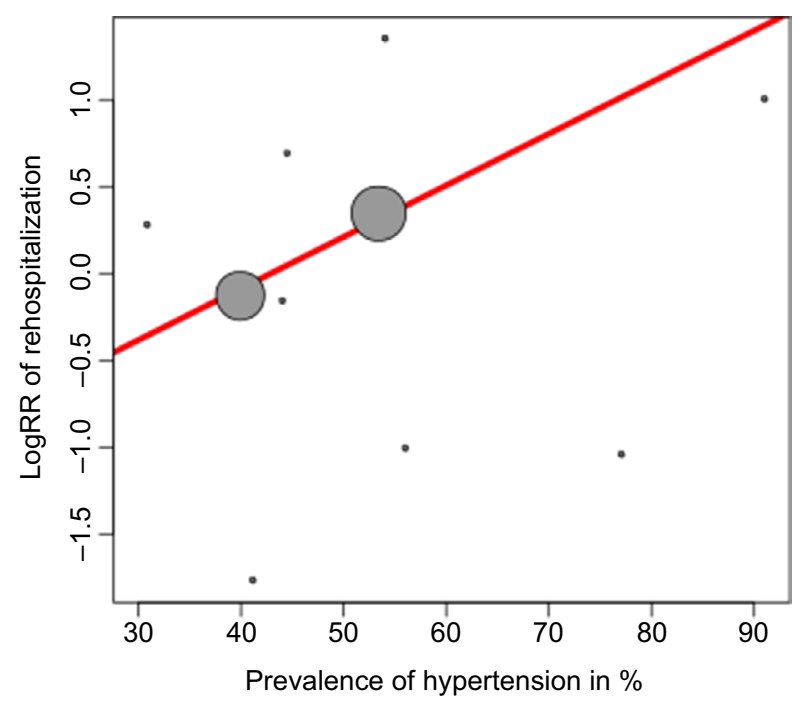

Figure 4 Meta-regression for the efficacy of ED compared to OD on the incidence of rehospitalization versus prevalence of hypertension.

Notes: The size of the circles is an indicator of the size of the trial. Data adapted from references 4, 13, 14, and 16-22.

Abbreviations: $E D$, early discharge; $O D$, ordinary discharge; $R R$, relative risk.

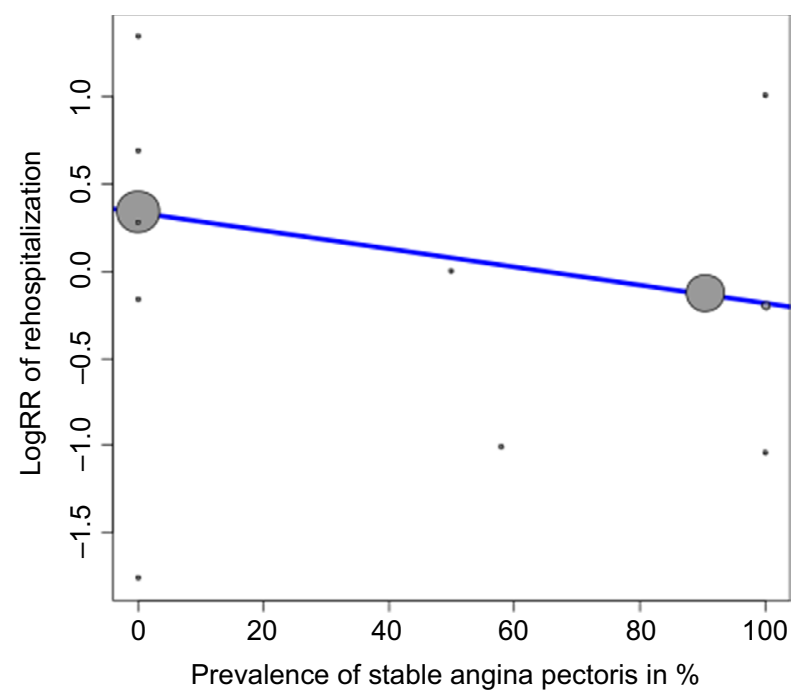

Figure 5 Meta-regression of the efficacy of ED compared to OD on the incidence of rehospitalization versus prevalence of stable angina pectoris

Notes: The size of the circles is an indicator of the size of the trial. Data adapted from references 4 and 13-23.

Abbreviations: $E D$, early discharge; $O D$, ordinary discharge; RR, relative risk.

\section{Cost}

We identified five RCTs that described the economic impact related to early discharge compared with ordinary discharge after PCI (Table 6). ${ }^{8,920,22,23}$ Most of the studies separated procedural cost and the cost of care and observation after the intervention. Three studies described total costs. Three articles mentioned only the cost of care and observation and estimated that procedural costs would be the same in both groups. One study calculated with larger procedural cost in the early discharge group due to the use of a vascular closure device. Rehospitalization within the follow-up period was included in the costs.

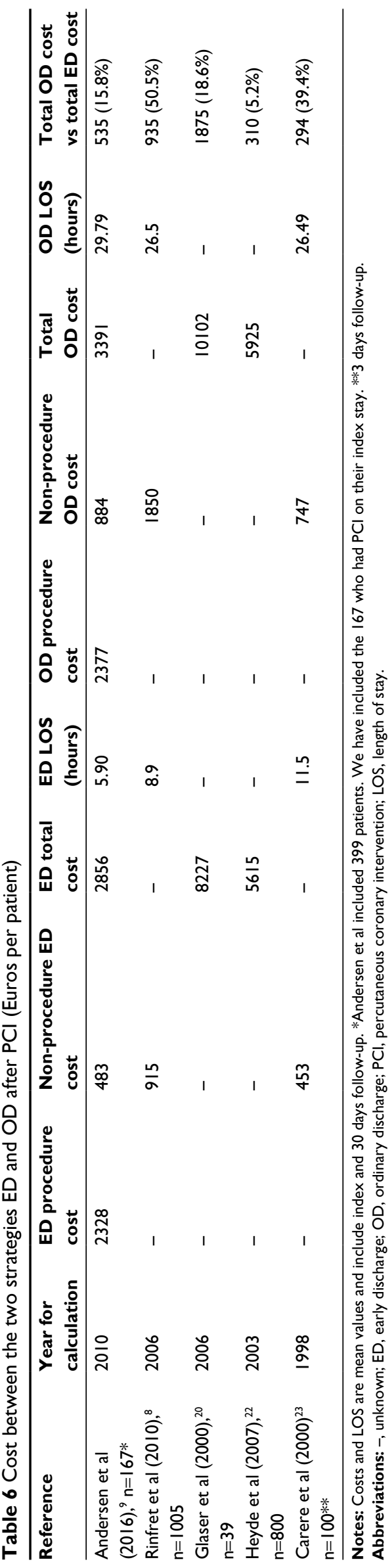


All studies found a decrease in total treatment cost for the early discharge group, with a reduction of $5.2 \%-50.1 \%$. In these studies, this means a saving of 294-1875 Euros per patient.

Three studies disclosed the length of stay (LOS). ${ }^{8,9,23}$ LOS included the index stay as well as subsequent rehospitalization within 30 days. Mean LOS varied between 5.9 hours in the early discharge group to 29.8 hours in the ordinary care group.

\section{Discussion}

\section{Safety}

The results of our systematic review indicated a reduction in the composite clinical endpoint with early discharge. However, there was a possibility of increased risk of rehospitalization for early discharge compared to ordinary care patients with hypertension and an increasing risk of $\mathrm{UAP} /$ revascularization with increasing age of the patient.

Our results regarding the safety of early discharge compared to ordinary care on the outcomes of the primary and secondary endpoints changed when we arranged the components of trial quality. The trials were assessed individually and methodologically, and their influence on effect size was explored. It has been shown that when studies of low methodological quality are incorporated into the meta-analysis, the estimate of quality can alter the interpretation of the benefit of the intervention. We found a reduction in the primary composite endpoint for the early discharge strategy. We found a possibility of increasing risk of rehospitalization with early discharge with increasing frequency of hypertension.

When heterogeneity was present, we performed metaregression analyses on study-level and patient-level variables. It was not performed if the number of studies was $<10$. We had specified patient-level variables a priori in the protocol of our meta-analysis. Only biologically plausible mechanisms were considered. ${ }^{28,29}$

Patient-related variables such as frequency of hypertension and age of patients can be subject to ecological fallacy and should be considered hypothesis generating, ${ }^{30}$ while the study-level variables that were considered in this metaregression (concealment of randomization, drop-out) can detect real associations between effect of the treatment and the study-level variables. ${ }^{31}$

Our results show that unstable patients are more often the subject of complications and rehospitalization. Increasing age often results in co-morbidities and a higher risk. An unexpected finding was that the prevalence of hypertension should mean increased risk for rehospitalization. This indicates that extra care must be taken to assess risk and in selecting the appropriate patients suitable for early discharge.

\section{Cost}

Procedural variables are the strongest predictor of postprocedural complications. ${ }^{32}$ Vavalle et $\mathrm{al}^{33}$ found that the patients with longer LOS often had more co-morbidities and inhospital complications. Analyzing procedural results can help triage patients suitable for early discharge. The use of validated risk scores such as Zwolle can be of help in identifying low-risk patients, suitable for early discharge. ${ }^{34}$ The decrease in costs in the early discharge group is due to the reduction in LOS, and thus in the cost of care, both at the index stay and in the days of follow-up. This is confirmed by Le Corvoisier et al. ${ }^{35}$

Bakhai et $\mathrm{al}^{3}$ found that the risk of a new clinical event within 1 year after ACS treated with PCI is high among the Western European population. A finding of Korsnes et al ${ }^{36}$ was that the first major adverse cardiovascular event is more costly than subsequent events. Several studies have found that most adverse events related to the intervention occur shortly post-procedure and not in an extended observational period. ${ }^{37-39}$ This is in line with the results of the studies included in this meta-analysis and also with our pooled results. This suggests that occurrence of adverse events is not related to the discharge procedure. It is thus possible to decrease direct expenses by reducing LOS, considering the specific diagnosis of the patient. Our results show an increased risk of rehospitalization in the early discharge group, with the exception for patients with stable angina. Despite this finding, we see a reduction of cost in the early discharge group.

The time allotted to an intervention is the same, regardless of discharge strategy. Early discharge does not free up resources in the laboratory itself. But by reduced LOS in the early discharge group, resources used for observation and monitoring of patients were made available. Unfortunately, none of the studies in our meta-analysis have explored in what way these additional resources have been utilized or in what way this affects the economy.

A limitation to this cost analysis is that not all studies disclose the total cost. The reporting of costs included is also of variable quality. We have looked at costs related to the population as a whole because the quantitative basis for differentiating between STEMI, NSTEMI, UAP, and elective procedures is too small. It is possible that cost-effectiveness may vary between the groups to a greater extent than shown here.

\section{Strengths and limitations}

The major limitations of our study were the use of heterogeneous patient populations, quality of the trials with respect 
to the effect estimated, heterogeneity, underpowered trials, and use of other endpoints than our primary endpoint and rehospitalization.

\section{Conclusion}

Expenses in the early discharge group were lower compared with the ordinary discharge group, mainly due to a reduction in LOS and the cost of care.

The pooled effect for the composite cardiovascular endpoint supports the safe use of early compared to ordinary discharge after PCI in the treatment of a heterogeneous population of patients with CAD. However, for the endpoint rehospitalization, an increased risk was noted in patients with STEMI, NSTEMI, or unstable angina, but not in patients with stable angina.

Clinical trials with homogeneous populations of ACS are needed to be conclusive on this issue.

\section{Disclosure}

The authors report no conflicts of interest in this work.

\section{References}

1. Cook S, Walker A, Hugli O, Togni M, Meier B. Percutaneous coronary interventions in Europe: prevalence, numerical estimates, and projections based on data up to 2004. Clin Res Cardiol. 2007;96(6):375-382.

2. Taylor MJ, Scuffham PA, McCollam PL, Newby DE. Acute coronary syndromes in Europe: 1-year costs and outcomes. Curr Med Res Opin. 2007;23(3):495-503.

3. Bakhai A, Ferrieres J, Iniguez A, et al. Clinical outcomes, resource use, and costs at 1 year in patients with acute coronary syndrome undergoing PCI: results from the Multinational APTOR Registry. J Interv Cardiol. 2012;25(1):19-27.

4. Knopf WD, Cohen-Bernstein C, Ryan J, Heselov K, Yarbrough N, Steahr G. Outpatient PTCA with same day discharge is safe and produces high patient satisfaction level. J Invasive Cardiol. 1999;11(5):290-295.

5. Laut KG, Foldspang A. The effects on length of stay of introducing a fast track patient pathway for myocardial infarction: a before and after evaluation. Health Serv Manage Res. 2012;25(1):31-34.

6. Do DH, Dalery K, Gervais A, et al. Same-day transfer of patients with unstable angina and non-ST segment elevation myocardial infarction back to their referring hospital after angioplasty. Can J Cardiol. 2006;22(5):405-409.

7. Vendrametto F, Oberhollenzer R, Pitscheider W. [Percutaneous coronary intervention and immediate re-transfer to the referring hospital for patients with acute coronary syndrome. A single-center experience]. G Ital Cardiol (Rome). 2006;7(4):281-286.

8. Rinfret S, Kennedy WA, Lachaine J, et al. Economic impact of same-day home discharge after uncomplicated transradial percutaneous coronary intervention and bolus-only abciximab regimen. JACC Cardiovasc Interv. 2010;3(10):1011-1019.

9. Andersen JG, Grepperud S, Klow NE, Johansen O. Effects on length of stay and costs with same-day retransfer to the referring hospitals for patients with acute coronary syndrome after angiography and/or percutaneous coronary intervention. Eur Heart J Acute Cardiovasc Care. 2016;5(4):375-380.

10. Popescu AM, Weintraub WS. Outpatient percutaneous coronary interventions hospital and health system costs saving while maintaining patient safety. JACC Cardiovasc Interv. 2010;3(10):1020-1021.
11. Brayton KM, Patel VG, Stave C, de Lemos JA, Kumbhani DJ. Same-day discharge after percutaneous coronary intervention: a meta-analysis. J Am Coll Cardiol. 2013;62(4):275-285.

12. Abdelaal E, Rao SV, Gilchrist IC, et al. Same-day discharge compared with overnight hospitalization after uncomplicated percutaneous coronary intervention: a systematic review and meta-analysis. JACC Cardiovasc Interv. 2013;6(2):99-112.

13. Azzalini L, Sole E, Sans J, et al. Feasibility and safety of an early discharge strategy after low-risk acute myocardial infarction treated with primary percutaneous coronary intervention: the EDAMI pilot trial. Cardiology. 2015;130(2):120-129.

14. Melberg T, Jorgensen M, Orn S, Solli T, Edland U, Dickstein K. Safety and health status following early discharge in patients with acute myocardial infarction treated with primary PCI: a randomized trial. Eur J Prev Cardiol. 2015;22(11):1427-1434.

15. Kim M, Muntner P, Sharma S, et al. Assessing patient-reported outcomes and preferences for same-day discharge after percutaneous coronary intervention: results from a pilot randomized, controlled trial. Circ Cardiovasc Qual Outcomes. 2013;6(2):186-192.

16. Andersen JG, Kløw N-E, Johansen O. Safe and feasible immediate retransfer of patients to the referring hospital after acute coronary angiography and percutaneous coronary angioplasty for patients with acute coronary syndrome. Eur Heart J Acute Cardiovasc Care. 2013;2(3): 256-261.

17. Falcone AM, Bose R, Stoler RC, et al. The AmBulatory Closure Device Percutaneous Intervention (ABCD-PCI) study: a single-center experience. Proc (Bayl Univ Med Cent). 2011;24(3):192-194.

18. Bertrand OF, De Larochelliere R, Rodes-Cabau J, et al. A randomized study comparing same-day home discharge and abciximab bolus only to overnight hospitalization and abciximab bolus and infusion after transradial coronary stent implantation. Circulation. 2006;114(24):2636-2643.

19. Kotowycz MA, Cosman TL, Tartaglia C, Afzal R, Syal RP, Natarajan MK. Safety and feasibility of early hospital discharge in ST-segment elevation myocardial infarction - a prospective and randomized trial in low-risk primary percutaneous coronary intervention patients (the Safe-Depart Trial). Am Heart J. 2010;159(1):e111-e116.

20. Glaser R, Gertz Z, Matthai WH, et al. Patient satisfaction is comparable to early discharge versus overnight observation after elective percutaneous coronary intervention. J Invasive Cardiol. 2009;21(9):464-467.

21. Jirmar R, Widimsky P, Capek J, Hlinomaz O, Groch L. Next day discharge after successful primary angioplasty for acute ST elevation myocardial infarction. An open randomized study "Prague-5". Int Heart J. 2008;49(6):653-659.

22. Heyde GS, Koch KT, de Winter RJ, et al. Randomized trial comparing same-day discharge with overnight hospital stay after percutaneous coronary intervention: results of the Elective PCI in Outpatient Study (EPOS). Circulation. 2007;115(17):2299-2306.

23. Carere RG, Webb JG, Buller CE, et al. Suture closure of femoral arterial puncture sites after coronary angioplasty followed by same-day discharge. Am Heart J. 2000;139(1 pt 1):52-58.

24. Abdelnoor M, Sandven I, Limalanathan S, Eritsland J. Postconditioning in ST-elevation myocardial infarction: a systematic review, critical appraisal, and meta-analysis of randomized clinical trials. Vasc Health Risk Manag. 2014;10:477-491.

25. Pogue J, Yusuf S. Overcoming the limitations of current meta-analysis of randomised controlled trials. Lancet. 1998;351(9095):47-52.

26. Wetterslev J, Thorlund K, Brok J, Gluud C. Trial sequential analysis may establish when firm evidence is reached in cumulative meta-analysis. $J$ Clin Epidemiol. 2008;61(1):64-75.

27. Moher D, Liberati A, Tetzlaff J, Altman DG, PRISMA Group. Preferred reporting items for systematic reviews and meta-analyses: the PRISMA statement. Ann Intern Med. 2009;151(4):264-269,W264.

28. Berlin JA, Santanna J, Schmid CH, Szczech LA, Feldman HI, AntiLymphocyte Antibody Induction Therapy Study Group. Individual patient- versus group-level data meta-regressions for the investigation of treatment effect modifiers: ecological bias rears its ugly head. Stat Med. 2002;21(3):371-387. 
29. Higgins JP, Green S. Cochrane Handbook for Systematic Reviews of Interventions. Vol. 5. Wiley Online Library; 2008. Available from: http://handbook.cochrane.org/chapter_9/9_6_4_meta_regression.htm. Accessed October 11, 2016.

30. Morgenstern H. Uses of ecologic analysis in epidemiologic research. Am J Public Health. 1982;72(12):1336-1344.

31. Schmid CH, Stark PC, Berlin JA, Landais P, Lau J. Meta-regression detected associations between heterogeneous treatment effects and studylevel, but not patient-level, factors. J Clin Epidemiol. 2004;57(7):683-697.

32. Koch KT, Piek JJ, Prins MH, et al. Triage of patients for short term observation after elective coronary angioplasty. Heart. 2000;83(5):557-563.

33. Vavalle JP, Lopes RD, Chen AY, et al. Hospital length of stay in patients with non-ST-segment elevation myocardial infarction. Am J Med. 2012;125(11):1085-1094.

34. Kotowycz MA, Syal RP, Afzal R, Natarajan MK. Can we improve length of hospitalization in ST elevation myocardial infarction patients treated with primary percutaneous coronary intervention? Can J Cardiol. 2009;25(10):585-588.
35. Le Corvoisier P, Gellen B, Lesault PF, et al. Ambulatory transradial percutaneous coronary intervention: a safe, effective, and cost-saving strategy. Catheter Cardiovasc Interv. 2013;81(1):15-23.

36. Korsnes JS, Davis KL, Ariely R, Bell CF, Mitra D. Health care resource utilization and costs associated with nonfatal major adverse cardiovascular events. J Manag Care Spec Pharm. 2015;21(6):443-450.

37. Jozic J, Orford J, Steinhubl S, Berger P, Hsu A, Topol E. Timing and correlates of very early major adverse clinical events following percutaneous coronary intervention. J Invasive Cardiol. 2008;20(3): $113-118$.

38. Thel MC, Califf RM, Tardiff BE, et al. Timing of and risk factors for myocardial ischemic events after percutaneous coronary intervention (IMPACT-II). Integrilin to minimize platelet aggregation and coronary thrombosis. Am J Cardiol. 2000;85(4):427-434.

39. Jabara R, Gadesam R, Pendyala L, et al. Ambulatory discharge after transradial coronary intervention: preliminary US single-center experience (Same-day TransRadial Intervention and Discharge Evaluation, the STRIDE Study). Am Heart J. 2008;156(6):1141-1146.
Vascular Health and Risk Management

\section{Publish your work in this journal}

Vascular Health and Risk Management is an international, peerreviewed journal of therapeutics and risk management, focusing on concise rapid reporting of clinical studies on the processes involved in the maintenance of vascular health; the monitoring, prevention and treatment of vascular disease and its sequelae; and the involvement of

\section{Dovepress}

metabolic disorders, particularly diabetes. This journal is indexed on PubMed Central and MedLine. The manuscript management system is completely online and includes a very quick and fair peer-review system, which is all easy to use. Visit http://www.dovepress.com/ testimonials.php to read real quotes from published authors. 